\title{
DOCUMENTO
}

\section{Rodulfo Amando Philippi, el naturalista de mayor aporte al conocimiento taxonómico de la diversidad biológica de Chile}

\author{
SERGIO A. CASTRO ${ }^{1 *}$, ARIEL CAMOUSSEIGHT² ${ }^{2}$, MÉLICA MUÑOZ-SCHICK ${ }^{2}$ \& FABIÁN M. JAKSIC ${ }^{1}$ \\ ${ }^{1}$ Centro de Estudios Avanzados en Ecología \& Biodiversidad, Pontificia Universidad Católica de Chile, Casilla 114-D, \\ Santiago, CP 6513677, Chile \\ ${ }^{2}$ Museo Nacional de Historia Natural de Santiago, Casilla 787, Santiago, Chile; \\ * Corresponding author: scastro@bio.puc.cl
}

\begin{abstract}
RESUMEN
Rodulfo Amando Philippi (1808-1904) es considerado uno de los naturalistas más influyentes en el desarrollo de las Ciencias Naturales en Chile. Entre las actividades científicas que desempeñó en el país está su dedicación al trabajo taxonómico y sistemático, que lo llevó a describir numerosas especies de plantas, animales y hongos. En este artículo se determina su contribución al conocimiento del patrimonio biológico de Chile, cuantificando el número y proporción de las especies actualmente válidas. De las especies descritas, 1.670 son consideradas válidas. Esta cifra equivale al 5,8\% de la diversidad biológica reconocida para Chile. $\mathrm{Su}$ mayor aporte se concentra en plantas vasculares, donde se encuentran vigentes 1.017 especies, que representan el $19 \%$ de la flora vascular de Chile. Mención especial requiere el caso de algunos grupos, tales como anfibios, fasmatódeos, dermápteros y dípteros, cuya actual diversidad incluye 14, 30, 16 y $9 \%$ de las especies de descritas por Philippi, respectivamente. De acuerdo a lo anterior y en comparación con otros taxónomos, Rodulfo Amando Philippi es el autor del mayor número de descripciones de especies válidas de la biota de Chile.
\end{abstract}

Palabras clave: Chile, historia de la ciencia, naturalistas, botánica, zoología.

\begin{abstract}
Rodulfo Amando Philippi (1808-1904) is considered one of most influential in the development of natural sciences in Chile. Among his scientific endeavors, he contributed taxonomic descriptions of the biological diversity of Chile, encompassing organisms in three living kingdoms: plants, animals and fungi. In this article we examine his contribution to the knowledge of the biological wealth of Chile, quantifying the number and proportion of current valid species he described. Our findings indicate that Philippi described 1,670 valid species, equivalent to $5.8 \%$ of the recognized biological diversity for Chile. His greatest contributions were in vascular plants with 1,017 species descriptions, which represent $19 \%$ of the vascular flora of Chile. Special mention is made regarding specific groups, such as amphibians, phasmids, dermapterans and dipterans, whose current diversity includes $14,30,16$ and $9 \%$ of species described by Philippi, respectively. In comparison to other taxonomists, R. A. Philippi is the author of the largest number of valid species in the biota of Chile.
\end{abstract}

Key words: Chile, botany, natural history, science history, zoology.

\section{INTRODUCCIÓN}

Chile presenta una larga tradición naturalista vinculada al estudio y descripción de las especies del territorio (Jaksic \& Lazo 1994, Simonetti et al. 1995), una de las expresiones más directas de la biodiversidad (May 1988, Diamond 1995). En efecto, las primeras iniciativas de corte netamente científico gestadas bajo esta orientación datan de principios del siglo XVIII, cuando el naturalista francés L. E. Feuilleé visitó el territorio entre 1709 y 1710 (Feuilleé 1714-1725, Philippi 1867). Feuilleé realizó observaciones científicas para la Corona española, y recolectó especies que posteriormente fueron estudiadas por botánicos y zoólogos europeos, incluyendo a C. Linneo (Linneo 1753, Looser 1953). 
Esfuerzos más sistemáticos y efectivos fueron realizados a partir de la instauración de la República (s. XIX), institución que procuró conocer los recursos naturales del país, y de paso institucionalizar la enseñanza de las Ciencias Naturales y la investigación científica (Serrano 1993, Saldivia 2003). En este contexto histórico, y no ajeno a razones políticas, económicas y sociales de la época, llegaron a Chile algunos naturalistas que contribuyeron a consolidar e institucionalizar las disciplinas científicas (Chardon 1949, Zizka 1992, Saldivia 2003), y a diferencia de los precedentes, generaron un conocimiento en historia natural de carácter local, que permaneció en el territorio y trascendió.

Rodulfo Amando Philippi (1808-1904) es uno de los más reconocidos e influyentes en el desarrollo de las Ciencias Naturales en Chile. Tras su llegada desde Alemania en 1851 a la edad de 43 años, Philippi rápidamente se incorporó a la incipiente escena académica y científica de nuestro país (Steenbuck 2003), y junto a su hijo Federico Philippi, desarrolló una de las carreras científicas de mayor impacto e importancia, cuyas contribuciones pueden apreciarse en diversas áreas de nuestro actual conocimiento científico (Muñoz-Schick 2003, Pequeño 2003, Castro \& Muñoz-Schick 2004).

Durante su permanencia en Chile, que se extendió hasta su muerte, Philippi participó de diversos cargos, entre los que se cuentan Director del Museo Nacional y profesor de Botánica y Zoología en la Universidad de Chile (Barros-Arana 1904, Gotschlich 1904). Además, publicó cerca de 400 artículos científicos en disciplinas tan disímiles como botánica, zoología, entomología, paleontología, geología, arqueología, climatología, biogeografía y educación (Barros-Arana 1904). Entre sus publicaciones se hallan los primeros textos de estudios de Ciencias Naturales publicados en Chile, dirigidos a estudiantes de Enseñanza Secundaria y Superior. Precisamente en ellos se esbozan intentos por introducir las ideas revolucionarias en la ciencia de la época, tales como la evolución orgánica (MárquezBreton 1982). En efecto, Philippi conoció las ideas "transformistas", y su inclusión en los textos docentes le causó serios inconvenientes ya que sin ser "partidario de tales doctrinas" fue sindicado como tal y ridiculizado (BarrosArana 1904, Márquez-Breton 1982). Para
Philippi era inconcebible aceptar la idea de transmutación de una especie en otra, como lo proponía el darwinismo, o de reiterados eventos de creación divina luego de sucesivas catástrofes geológicas, como lo proponía Cuvier (Philippi 1885, p. 370-371). Aunque en sus publicaciones acepta explícitamente que las especies se originaron por creación divina (Philippi 1869, p. 2), otros de sus escritos admiten la posibilidad de la modificación morfológica heredable (adaptación). Es así que establece que las plantas comunes entre Chile y Europa se halla un grupo que responde a la siguiente condición: "que en diferentes partes del globo, cuando las condiciones de la vida para los vejetales han sido idénticas o casi idénticas, han nacido especies idénticas o mui semejantes, i que estas especies no han peregrinado ni han sido trasportadas por casualidad de un punto a otro" (Philippi 1875, p. 140).

Uno de los aspectos más notables de la actividad científica de Philippi fue su labor como descriptor de la riqueza biológica de Chile (Zizka 1992, Marticorena 1995, Pequeño 2003, Castro \& Muñoz 2004). Las numerosas expediciones que realizó a lo largo del país (Taylor \& Muñoz-Schick 1994) le permitieron colectar suficiente material para realizar contribuciones en el campo taxonómico, contando con la ayuda de colaboradores que le remitieron permanentemente material de estudio de diversos taxa y localidades (Gotschlich 1904). Sin embargo, con el tiempo numerosas especies que describió fueron sinonimizadas o invalidadas como resultado de la reinterpretación de la variación fenotípica y el abandono de la concepción tipológica de especie (Marticorena 1995, p. 35), así como por la aplicación de las reglas de prioridad de los códigos nomenclaturales para aquellas especies que habían sido descritas previamente (Castro datos no publicados).

En 1902, la figura de Philippi había sido incluida en la "Galería de Naturalistas de Chile" (Porter 1902), una sección de la Revista Chilena de Historia Natural dedicada al reconocimiento de los investigadores y sus aportes al conocimiento científico. Al deceso de Philippi en julio de 1904, Porter (1904) escribió un breve obituario en esta misma revista, destacando que entre el legado de Philippi se hallan numerosas especies que pudo describir en 53 años de actividad científica ininterrumpida. Su extenso 
trabajo en taxonomía planteó inmediatamente la inquietud de cuantificar su aporte científico, desafío propuesto por sus dos biógrafos, quienes carecían de formación biológica necesaria para realizar esta tarea (Barros-Arana 1904, Gotschlich 1904).

A 100 años de su fallecimiento, centramos nuestro esfuerzo en determinar la contribución de R. A. Philippi al conocimiento de nuestra biodiversidad. Específicamente, realizamos un catastro de las especies que describió y que actualmente son reconocidas como entidades taxonómicas válidas, y determinamos la representación de estos taxa en la riqueza biológica de cada grupo. Con ello intentamos dar relevancia a un naturalista del siglo XIX, cuyo aporte es necesario resaltar a un siglo de su desaparición.

\section{MATERIALES Y MÉTODOS}

Revisamos las publicaciones de R. A. Philippi e identificamos los grupos biológicos en que trabajó, a niveles de Phyllum, Clase, Orden y Familia. Con esta información examinamos catálogos actualizados, listas sistemáticas y estudios recientes para cada uno de estos grupos taxonómicos, registrando el nombre y número de las especies atribuidas a R. A. Philippi, así como el año de cada descripción. Algunas de estas (especialmente en plantas e insectos) fueron publicadas en más de una ocasión por lo que solo consideramos aquella más temprana como descripción original. Adicionalmente, registramos la posición sistemática de cada especie de acuerdo al ordenamiento actual proporcionado por cada fuente.

\section{Las especies de Philippi}

Philippi describió numerosas especies en Plantae (Muñoz-Pizarro 1960), lo que nos llevó a revisar catálogos de musgos (He 1998), pteridofitas y gimnospermas (Marticorena \& Rodríguez 1995), y angiospermas (Marticorena \& Quezada 1985, con modificaciones indicadas en Marticorena \& Quezada 1987, Hunt 1999, Marticorena \& Rodríguez 2001), publicados para Chile. La información para hongos Basidiomycetes, Ficomycetes, Ascomycetes y Deuteromycetes -considerados plantas en el siglo XIX - fue obtenida de Mujica \& Vergara (1980) y Lazo (2001).

En el caso de los vertebrados, recopilamos la información disponible para peces (Pequeño 1989, 1997), anfibios (Veloso \& Navarro 1988), reptiles (Veloso \& Navarro 1988, con las modificaciones sugeridas por Núñez \& Jaksic 1992), aves (Araya et al. 1995), y mamíferos (Tamayo et al. 1987, con las indicaciones de Yáñez \& Muñoz-Pedreros 2000).

Para invertebrados, reunimos la información de cnidarios (Philippi 1892, Moyano 1995), bryozoos (Moyano 1991) y moluscos (Valdovinos-Zarges 1999). En crustáceos, revisamos catálogos y listas de especies a nivel de orden (Cladocera: Ruiz \& Bahamonde 1989; Calanoida: Ruiz \& Bahamonde 1989; Harpacticoida: Ruiz \& Bahamonde 1989; Cyclopoida: Ruiz \& Bahamonde 1989; Bathynellacea: Báez com. pers.; Amphipoda: González 2003; Isopoda: Menzies 1961, González \& Thiel 2004; Decapoda: Retamal 1981), y en ausencia de catálogos o listas sistemáticas consultamos directamente a un especialista (Mystacocarioida, Rhizocephala y Stomatopoda: Pedro Báez com. pers.). Los equinodermos (clases Stelleroidea, Echinoidea, Holoturoidea y Crinoidea presentes en Chile) fueron recopilados de Larraín (1995). Los insectos fueron obtenidos a partir de la revisión de las publicaciones originales de Philippi respecto de este grupo (véase Apéndice 1), conformándose un catálogo de especies que fue actualizado nomenclaturalmente (a nivel de género, familia y orden), y cuyo estatus taxonómico fue verificado por la revisión catálogos y publicaciones taxonómicas recientes.

\section{Análisis}

Para estimar cuantitativamente el aporte de Philippi en la descripción taxonómica chilena, calculamos la fracción porcentual de especies descritas por este naturalista en cada grupo a nivel de clase u orden. En el caso de los insectos, este cálculo se realizó en base a los últimos recuentos de cada uno de los órdenes involucrados, a pesar que algunos de ellos solo son aproximaciones estimadas (Blattariae: Moroni \& Camousseight 1976, Coleoptera: Elgueta 2000, Dermaptera: Reichardt 1968a,b, Diplura: Camousseight 1995a, Diptera: González 1995, Hemiptera: Prado 1995, 
Hymenoptera: Elgueta \& Rojas 2000, Lepidoptera: Parra 1995, Mantodea: Camousseight 1995b, Orthoptera: Elgueta et al. 1999, Camousseight \& Carbonell 1999, Phasmatodea: Camousseight 1995c).

Adicionalmente, analizamos el tipo de autoría y la cronología de las descripciones realizadas por Philippi. En el caso de la autoría distinguimos descripciones originales con y sin modificaciones nomenclaturales posteriores, descripción en coautorías con y sin modificaciones nomenclaturales posteriores, y descripciones que le atribuyen otros autores. Adicionamos una nómina y frecuencia de taxa en incertae sedis. Por otra parte, el análisis cronológico de sus descripciones vigentes nos permitió tener una idea complementaria al esfuerzo de trabajo invertido por el naturalista en los distintos grupos taxonómicos con los que trabajó, distinguiendo plantas (incluyendo hongos), invertebrados y vertebrados.

\section{RESULTADOS}

En nuestra revisión, registramos 1.670 especies descritas por Philippi que son consideradas válidas en la actualidad. De ellas, 1.017 especies son plantas, 650 son animales y tres son hongos (Tabla 1). En el caso de las plantas, el mayor número de contribuciones se encontró en angiospermas $(\mathrm{n}=1.006)$ y pteridófitas $(\mathrm{n}=$ 163), mientras que para el reino animal hallamos 602 invertebrados y 48 vertebrados. Entre los primeros hay 525 insectos (principalmente dípteros y coleópteros; Tabla 1), 59 moluscos, cinco cnidarios (todos anthozoos), cuatro crustáceos. Los vertebrados corresponden a 22 especies de aves, nueve de peces, siete de mamíferos, seis de anfibios y cuatro de reptiles. La proporción de especies descritas por Philippi en las distintas clases u órdenes en que trabajó varía entre 0,09 y $31 \%$ (Tabla 1 ). En total, estas descripciones equivalen a $7,1 \%$ de la riqueza específica actualmente reconocida para estos taxa. Desde el punto de vista cuantitativo, es importante notar que la contribución de este autor en angiospermas, anfibios, dermápteros y fasmatódeos supera el $10 \%$ de las especies reconocidas (Tabla 1 ).

De las 1.670 especies actualmente válidas descritas por Philippi, $59 \%$ conserva la nominación original propuesta por el naturalista, mientras que $39,3 \%$ ha experimentado modificaciones nomenclaturales a nivel de género o familia (Tabla 1). Una fracción menor de especies se halla en incertae sedis $(<0.1 \%)$, debido a la falta de material tipo y/o vagas descripciones que no permiten establecer la validez taxonómica de dichas especies. En términos de autorías y coautorías, Philippi publicó la mayor parte de sus descripciones como único autor ( $89 \%$ de los casos), y siempre en primer lugar. Solo en el $11 \%$ de sus descripciones con vigencia actual Philippi incorporó a otro descriptor, que en la mayor parte de los casos fue su hijo Federico; en tanto que L. Landbeck aparece como coautor en descripciones de un mamífero y diez aves, mientras que C. Juliet participó como coautor en la descripción de una especie de coleóptero.

De acuerdo a la cronología de las descripciones de especies actualmente vigentes, el trabajo botánico de R. A. Philippi se realizó entre 1855 y 1904, con períodos más intensos de actividad entre 1855 y 1857 , y entre 1890 y 1898 (Fig. 1A). Su labor en vertebrados también se extendió ampliamente durante la segunda mitad del siglo XIX (entre 1853 y 1903), aunque involucrando un menor número de especies (Fig. 1B). En contraste, la descripción de invertebrados puede ser caracterizada como episódica, ya que su mayor productividad descriptiva se realizó en un solo período de tres años: entre 1863 y 1866 (Fig. 1C). Es interesante notar que la descripción de invertebrados chilenos la inició Philippi en 1844, año en que aún residía en Europa.

\section{DISCUSIÓN}

Previo a la llegada de Philippi a Chile, dos notables naturalistas habían realizado notables avances en el conocimiento del patrimonio biológico del país. Por una parte, Juan Ignacio Molina, quien publicó numerosas especies nuevas para la ciencia (Molina 1782), y por otra, Claudio Gay (1845-1854), quien junto a su equipo de trabajo compendió la información disponible para Chile hasta la primera mitad del siglo XIX, al mismo tiempo de realizar nuevas descripciones. La labor de estos naturalistas precedentes sirvió como un importante sustrato para el trabajo de Philippi (Saldivia 2003). 
TABLA 1

Número de especies válidas descritas por R.A. Philippi, y su proporción respecto de la diversidad de cada grupo en Chile

Number of valid species described by R.A. Philippi, and their proportion with respect to the diversity of each group in Chile

\begin{tabular}{|c|c|c|c|c|c|c|c|c|}
\hline $\begin{array}{l}\text { Categorías } \\
\text { taxonómicas }\end{array}$ & Philippi & (Philippi) & $\begin{array}{l}\text { Philippi } \\
\text { et al. }\end{array}$ & $\begin{array}{r}\text { (Philippi } \\
\text { et al.) }\end{array}$ & $\begin{array}{r}\text { Philippi } \\
\text { ex }\end{array}$ & $\begin{array}{r}\text { Total } \\
\text { Philippi }\end{array}$ & $\begin{array}{l}\text { Total } \\
\text { Taxa }\end{array}$ & $\%$ \\
\hline \multicolumn{9}{|l|}{ Plantas } \\
\hline Angiospermas & 727 & 255 & 0 & 0 & 24 & 1.006 & 5.070 & 19,8 \\
\hline Gimnospermas & 2 & 0 & 0 & 0 & 2 & 4 & 16 & 25,0 \\
\hline Pteridofitas & 6 & 1 & 0 & 0 & 0 & 7 & 163 & 4,2 \\
\hline \multicolumn{9}{|l|}{ Animales } \\
\hline Mamíferos & 2 & 4 & 0 & 1 & 0 & 7 & 148 & 4,7 \\
\hline Aves & 5 & 0 & 17 & 0 & 0 & 22 & 527 & 4,1 \\
\hline Reptiles & 0 & 4 & 0 & 0 & 0 & 4 & 97 & 4,1 \\
\hline Anfibios & 1 & 5 & 0 & 0 & 0 & 6 & 41 & 14,6 \\
\hline Octeistios & 1 & 1 & 0 & 0 & 0 & 2 & 700 & 0,2 \\
\hline Congrictios & 0 & 7 & 0 & 0 & 0 & 7 & 430 & 1,6 \\
\hline \multicolumn{9}{|l|}{ Cnidarios } \\
\hline Antozoos & 4 & 1 & 0 & 0 & 0 & 5 & 176 & 2,8 \\
\hline Hidrozoos & 0 & 0 & 0 & 0 & 0 & 0 & 141 & 0,0 \\
\hline \multicolumn{9}{|l|}{ Moluscos } \\
\hline Caudofoveatos & 0 & 0 & 0 & 0 & 0 & 0 & 7 & 0,0 \\
\hline Solenogastros & 0 & 0 & 0 & 0 & 0 & 0 & 5 & 0,0 \\
\hline Placóforos & 0 & 0 & 0 & 0 & 0 & 0 & 62 & 0,0 \\
\hline Gastrópodos & 17 & 26 & 0 & 0 & 0 & 43 & 873 & 4,9 \\
\hline Bivalvos & 7 & 9 & 0 & 0 & 0 & 16 & 232 & 6,8 \\
\hline Escafópodos & 0 & 0 & 0 & 0 & 0 & 0 & 7 & 0,0 \\
\hline Cefalópodos & 0 & 0 & 0 & 0 & 0 & 0 & 92 & 0,0 \\
\hline \multicolumn{9}{|l|}{$\begin{array}{l}\text { Crustáceos } \\
\text { Branquiópodos }\end{array}$} \\
\hline Orden Cladóceros & 0 & 0 & 0 & 0 & 0 & 0 & 49 & 0,0 \\
\hline \multicolumn{9}{|l|}{ Maxilópodos } \\
\hline Orden Mistacocarioideos & 0 & 0 & 0 & 0 & 0 & 0 & 1 & 0,0 \\
\hline Orden Rizocéfalos & 0 & 0 & 0 & 0 & 0 & 0 & 4 & 0,0 \\
\hline Orden Calanoideos & 0 & 0 & 0 & 0 & 0 & 0 & 23 & 0,0 \\
\hline Orden Harpacticoideos & 0 & 0 & 0 & 0 & 0 & 0 & 23 & 0,0 \\
\hline Orden Ciclopoideos & 0 & 0 & 0 & 0 & 0 & 0 & 22 & 0,0 \\
\hline \multicolumn{9}{|l|}{ Malacostracos } \\
\hline Orden Estomatópodos & 0 & 0 & 0 & 0 & 0 & 0 & 4 & 0,0 \\
\hline Orden Batinelaceos & 0 & 0 & 0 & 0 & 0 & 0 & 12 & 0,0 \\
\hline Orden Anfípodos & 0 & 1 & 0 & 0 & 0 & 1 & 185 & 0,5 \\
\hline Orden Isópodos & 0 & 0 & 0 & 0 & 0 & 0 & 90 & 0,0 \\
\hline Orden Decápodos & 0 & 3 & 0 & 0 & 0 & 3 & 203 & 1,4 \\
\hline \multicolumn{9}{|l|}{ Equinodermos } \\
\hline Clase Crinoideos & 0 & 0 & 0 & 0 & 0 & 0 & 14 & 0,0 \\
\hline Clase Esteleroideos & 0 & 6 & 0 & 0 & 0 & 6 & 198 & 3,0 \\
\hline Clase Equinoideos & 0 & 2 & 0 & 0 & 0 & 2 & 64 & 3,1 \\
\hline Clase Holoturoideos & 0 & 1 & 0 & 0 & 0 & 1 & 74 & 1,3 \\
\hline \multicolumn{9}{|l|}{ Insectos } \\
\hline Blattarios & 0 & 1 & 0 & 0 & 0 & 1 & 26 & 3,8 \\
\hline Coleópteros & 33 & 15 & 55 & 70 & 0 & 173 & 3.947 & 4,3 \\
\hline Dermápteros & 0 & 2 & 0 & 0 & 0 & 2 & 12 & 16,6 \\
\hline Dipluros & 0 & 1 & 0 & 0 & 0 & 1 & 22 & 4,5 \\
\hline Dípteros & 88 & 213 & 0 & 0 & 0 & 293 & 2.996 & 9,8 \\
\hline Hemípteros & 1 & 3 & 0 & 0 & 0 & 4 & 277 & 1,4 \\
\hline Himenópteros & 6 & 7 & 0 & 0 & 0 & 13 & 1.411 & 0,9 \\
\hline Lepidópteros & 7 & 17 & 0 & 0 & 0 & 24 & 1.327 & 1,8 \\
\hline Mantodeos & 0 & 0 & 0 & 0 & 0 & 0 & 2 & 0,0 \\
\hline Ortópteros & 3 & 7 & 0 & 0 & 0 & 10 & 145 & 6,8 \\
\hline Fasmatodeos & 2 & 2 & 0 & 0 & 0 & 4 & 13 & 30,7 \\
\hline Hongos & 3.300 & $<0,1$ & & & & & & \\
\hline Basidiomicetos & 1 & 0 & 0 & 0 & 1 & 2 & & \\
\hline Ficomicetos & 0 & 0 & 0 & 0 & 0 & 0 & & \\
\hline Ascomicetos & 1 & 0 & 0 & 0 & 0 & 1 & & \\
\hline Deuteromicetos & 0 & 0 & 0 & 0 & 0 & 0 & & \\
\hline Total & 915 & 586 & 72 & 71 & 26 & 1.670 & 23.231 & 7,1 \\
\hline
\end{tabular}



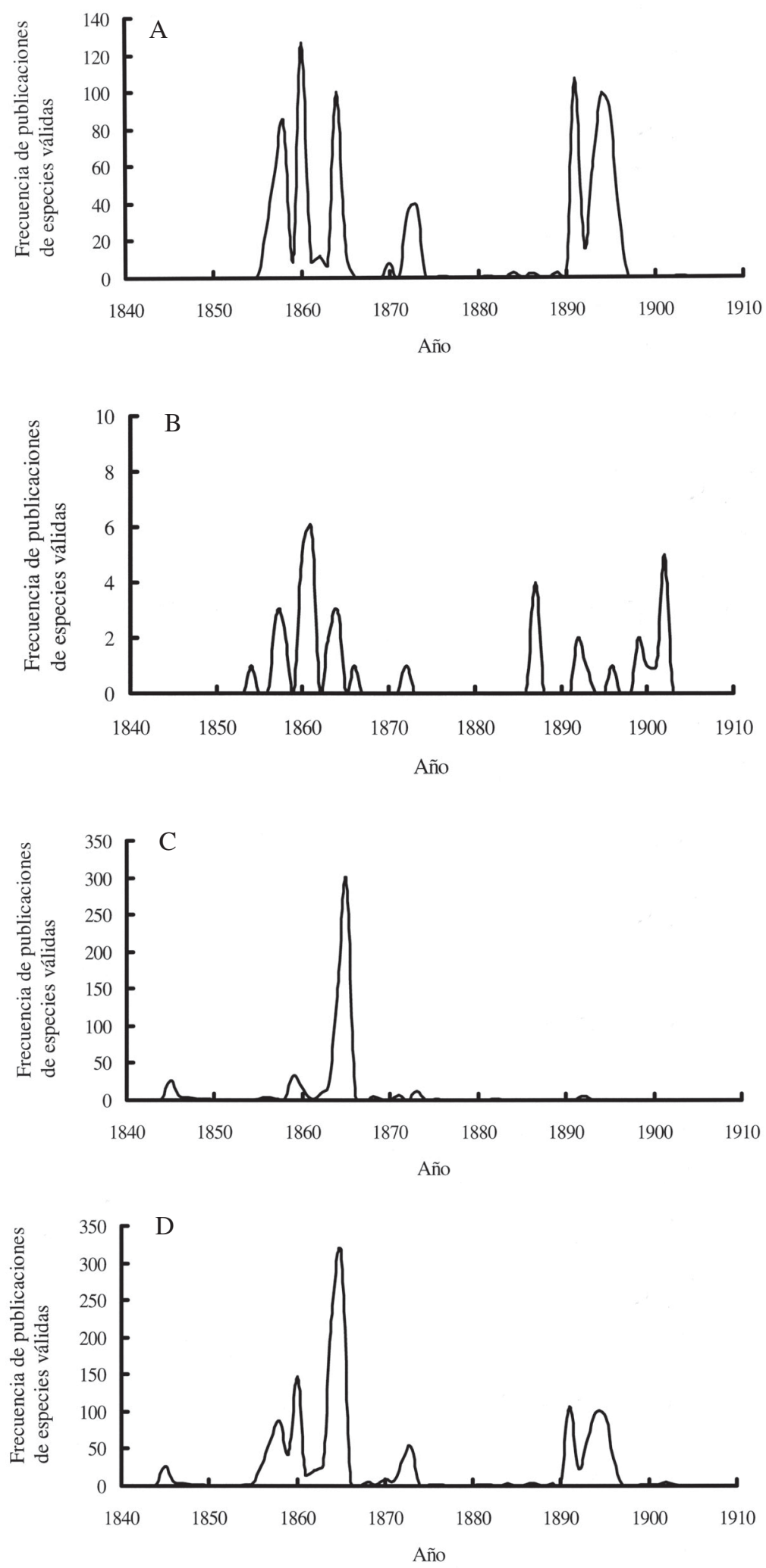

Fig. 1: Cronología de las publicaciones de las especies actualmente válidas descritas por R. A. Philippi. A: plantas; B: vertebrados; C: invertebrados; D: todos.

Chronology of the currently valid species descriptions by R. A. Philippi. A: plants; B: vertebrates; C: invertebrates; D: all. 
Reconociéndose para Chile una riqueza de 28.508 especies (Simonetti et al. 1995, p. 362), Philippi contribuyó a la descripción de 1.670, equivalente a $5,8 \%$ de esta diversidad. Este valor aumenta a $7,2 \%$ si se consideran solo aquellos grupos taxonómicos en que efectivamente trabajó. Nuestros resultados permiten apreciar cuantitativamente que el aporte de Philippi se concentró en plantas vasculares, donde se hallan vigentes 1.017 de sus descripciones originales, las que representan $19 \%$ de las plantas vasculares reconocidas para Chile. Mención especial requiere el caso de anfibios, fasmatódeos y dermápteros, cuya actual diversidad incluye $>10 \%$ de especies descritas originalmente por Philippi, respectivamente.

Pese a la amplia diversidad de tipos de organismos que este naturalista estudió en Chile, su contribución en botánica es la más significativa, no solo por el número de especies descritas sino por la representación respecto de la riqueza reconocible en el territorio. Ningún otro naturalista o botánico ha sido tan prolífico en la descripción de plantas chilenas. Castro \& Muñoz-Schick (2004) mostraron que unos 600 autores han realizado la descripción de la flora vascular chilena; de ellos, Philippi fue el más productivo, seguido por los ingleses W. J. Hooker y G. Arnott que en coautoría (H. et A.) describieron 169 plantas presentes en Chile. En la actualidad, a otros reconocidos naturalistas tales como C. Gay y J. I. Molina se les adscribe la autoría de 77 y 33 especies de plantas, respectivamente (Castro \& Muñoz-Schick 2004). De acuerdo a la cronología de la descripción de especies válidas, Philippi desarrolló esfuerzos continuos y persistentes en el estudio de la flora chilena, mientras que la descripción de invertebrados y hongos recibió atención episódica. Por ejemplo, $96 \%$ de los insectos considerados válidos para Chile los describió entre 1859 y 1866; $66 \%$ de los moluscos considerados válidos para Chile los describió entre 1841 y 1849.

El trabajo de Philippi en invertebrados le permitió describir 603 especies actualmente consideradas válidas. La mayor parte de los invertebrados estudiados por este naturalista fueron insectos, cuyo material tipo se encuentra depositado en el Museo Nacional de Historia Natural (Mostny-Glaser \& Niemeyer-Fernández 1983). Es interesante notar que Philippi siempre manifestó especial atención por la entomofauna (Pequeño 2003) ${ }^{1}$; sin embargo, en numerosas oportunidades observó que la diversidad de insectos era bastante pobre en Chile comparada con las regiones templadas del hemisferio norte (Philippi 1862). Un hecho desconcertante es que en la primera mitad del siglo XIX Philippi gozaba de un reconocido prestigio mundial como malacólogo (Steenbuck 2003) ${ }^{2}$, habiendo trabajado alrededor de nueve años en sistemática y taxonomía de moluscos (Barros-Arana 1904, pp. 229-230), pero tras su llegada a Chile no le dio continuidad a esta trayectoria. En la actualidad, 59 de sus descripciones de moluscos son considerados válidas, de los cuales solo 20 taxa fueron descritos desde Chile, mientras que 39 fueron descritos entre 1844-1851, cuando el autor aún residía en Alemania ${ }^{3}$.

De los vertebrados descritos por Philippi solo 41 especies son actualmente válidas. Igual que en plantas, la dedicación al estudio de vertebrados también tuvo una amplia extensión temporal. Philippi se concentró principalmente en aves (15 especies), de las cuales seis fueron colectadas en el desierto de Atacama y dos en el archipiélago Juan Fernández (Philippi 1902). Los ejemplares tipo se encuentran depositados en el Museo Nacional de Historia Natural (Pequeño 2003).

Ciertamente, Philippi publicó un enorme número de descripciones de especies, pero la mayor parte de ellas han sido invalidadas. En plantas, por ejemplo, describió cerca de 3.700 especies (Muñoz-Pizarro 1960), de las que solo se encuentra vigente $33 \%$; en insectos describió 808 especies, de las cuales se encuentra vigente $66 \%$. Por consiguiente, el aporte cuantitativo de Philippi al conocimiento de nuestra riqueza biológica podría modificarse levemente a la luz de futuras revisiones

\footnotetext{
${ }^{1}$ Philippi realizó su memoria en ortópteros: Philippi RA (1830) Orthoptera berolinensia. Inaugural dissertation. Universidad de Berlín.

2 Diversos taxónomos europeos le dedicaron especies, entre ellos Huppé, el malacólogo que revisó los moluscos en la obra de C. Gay (Historia Física y Política de Chile), quien le dedicó Diplodonta philippi.

3 Es un hecho poco conocido que Philippi describió algunas especies de moluscos y una especie de equinodermo echinoideo antes de llegar a Chile. Estos pertenecen principalmente a la región de Magallanes, los que en 1843 fueron colectados y enviados por su hermano Bernardo tras participar de una expedición político-militar a dicha región.
} 
sistemáticas y taxonómicas, especialmente en invertebrados cuya diversidad se encuentra pobremente estudiada (Lozada \& Solervicens 1995). Se ha sugerido que la concepción tipológica de especie que prevaleció entre los naturalistas del siglo XIX dio estatus de especie a variaciones fenotípicas de un taxón (Saldivia 2003). Adicionalmente, es posible argumentar que el limitado acceso a publicaciones y ejemplares depositados en otros museos, indujo la publicación de descripciones de especies en desconocimiento que ya habían sido descritas.

La mayor parte de las publicaciones de Philippi fueron realizadas como único autor. No obstante, mantuvo contacto con connotados naturalistas de la época, tales como A. von Humboldt, C. Darwin y F. Ameghino (Gotschlich 1904). Hasta qué punto estas relaciones influyeron su investigación es materia de discusión y futuro análisis. Philippi rechazaba las ideas darwinianas (Philippi 1885), aunque su argumentación sugiere que no las conocía a cabalidad (Philippi 1875, p. 140). De hecho, su extensa biblioteca personal carecía de una copia del texto "Sobre el Origen de las Especies" (véase Anónimo 1911). Hacia el final de su vida le dictó a su secretario personal lo siguiente: "Esponjas i Polythalamios fósiles se encuentran también en el período de transición, i no se han perfeccionado hasta el día de hoy, como lo exige la teoría; pero al lado de estos se encuentran también los restos de animales que tienen una organización mas alta. Así se encuentran el mismo tiempo numerosos restos de corales, i esto de jéneros que todavía hoy existen, como Gorgania i Astrea. Tampoco estos jéneros se han perfeccionado o evolucionado en el transcurso de los millares de años. Los trilobites, que entonces vivían en grandes cantidades, han desaparecido también sin que de ellos se haya desarrollado otro jénero de animales" (Gotschlich 1904). Aparentemente, la inconsistencia que observa Philippi en la teoría darwiniana radica en su experiencia directa con el material fósil. Sus agudas observaciones respecto de la constancia morfológica en algunos grupos taxonómicos (moluscos, helechos, coníferas) en el registro paleontológico constituye una de las principales líneas de sustento que darían origen a la Teoría de Equilibrio Puntuado formulada por Eldredge \& Gould (1972), 70 años después.
La actividad naturalista de Philippi en Chile estuvo estrechamente ligada al Museo Nacional de Historia Natural, institución de la cual fue Director. Por esta razón, sus colectas y material tipo fueron depositadas ahí. En la actualidad, el estado de estas colecciones es heterogéneo (Muñoz-Pizarro 1960, Mostny-Glaser \& Niemeyer-Fernández 1983, Letelier et al. 2003), hecho lamentable si se considera su importancia como sustrato biológico para estudios en sistemática, taxonomía, ecología, macro y microevolución, biogeografía y macroecología (Sigüeza \& Llorente-Bousquets 1994, Lane 1996, Shaffer et al. 1998). A esto se adiciona el escaso interés por consultar este material por parte de los actuales investigadores naturalistas.

Pese a lo voluminoso y extenso del trabajo de Philippi en taxonomía, su aporte puede ser ampliado aún más, al considerar la descripción de categorías supra e infraespecíficas (variedades, géneros, familias nuevas para la ciencia), así como de especies fósiles (vertebrados e invertebrados). Aunque este tipo de descripciones no fueron incorporadas en el presente estudio, con la información disponible y en comparación a otros taxónomos podemos concluir que R. A. Philippi es el autor que ha realizado el mayor número de descripciones taxonómicas de nuestra diversidad biológica actual.

\section{AGRADECIMIENTOS}

Los autores agradecen los comentarios e información dispuesta por Pedro Báez (Museo Nacional de Historia Natural), Nibaldo Bahamonde (Museo Nacional de Historia Natural), Jorge Basly (coleccionista particular), Javier Figueroa (Pontificia Universidad Católica de Chile), Óscar Gálvez (Museo Nacional de Historia Natural), Exequiel González (Universidad del Norte), Sergio Letelier (Museo Nacional de Historia Natural), Erika Mutschke (Universidad de Magallanes), María Eliana Ramírez (Museo Nacional de Historia Natural), Nicolás Rozbaczylo (Pontificia Universidad Católica de Chile), Cecilia Osorio (Universidad de Chile), Juan Carlos Torres-Mura (Museo Nacional de Historia Natural) y Patricio Zavala (Pontificia Universidad Católica de Chile). Inés Meza, Luis Hidalgo y Leonardo Matus colaboraron con la búsqueda de material bibliográfico. 


\section{LITERATURA CITADA}

ANÓNIMO (1911) Inventario de la "Biblioteca Philippi", adquirida por el Museo Nacional. Boletín del Museo Nacional de Chile 3: 469-551.

ARAYA B, M BERNAL, R SCHLATTER \& M SALABERRY (1995) Lista patrón de las aves chilenas. Tercera edición. Editorial Universitaria, Santiago. 35 pp.

BARROS-ARANA D (1904) El doctor don Rodolfo Amando Philippi, su vida i sus obras. Santiago. Imprenta Cervantes, Santiago, Chile. 248 pp.

CAMOUSSEIGHT A (1995a) Diplura. En: Simonetti JA, MT Kalin-Arroyo, AE Spotorno \& E Lozada (eds) Diversidad biológica de Chile: 211-213. Comisión Nacional de Investigación Científica y Tecnológica, Santiago, Chile.

CAMOUSSEIGHT A (1995b) Mantodea, Embrioptera, Raphidioptera y Strepsiptera. En: Simonetti JA, MT Kalin-Arroyo, AE Spotorno \& E Lozada (eds) Diversidad biológica de Chile: 227-229. Comisión Nacional de Investigación Científica y Tecnológica, Santiago, Chile.

CAMOUSSEIGHT A (1995c) Phasmodea. En: Simonetti JA, MT Kalin-Arroyo, AE Spotorno \& E Lozada (eds) Diversidad biológica de Chile: 230-233. Comisión Nacional de Investigación Científica y Tecnológica, Santiago, Chile.

CASTRO SA \& M MUÑOZ-SCHICK (2004) Naturalistas y botánicos: una estimación y caracterización de su aporte al conocimiento de la diversidad florística de Chile. Boletín Museo Nacional de Historia Natural 53: 7-16.

CHARDON CE (1949) Los naturalistas en la América Latina. Editora del Caribe, Ciudad Trujillo, República Dominicana. 386 pp.

DIAMOND JM (1995) How many species are yet to be described? Nature 315: 538-539.

ELDREDGE N \& SJ GOULD (1972) Punctuated equilibria: an alternative to phyletic gradualism. En: Schopf TJM (ed) Models in paleobiology: 82-115. Freeman, Cooper \& Co. San Francisco, California, USA.

ELGUETA M \& F ROJAS (2000) Hymenoptera de Chile. En: Martín-Piera F, JJ Morrone \& A Melic (eds) Hacia un proyecto CYTED. PrIBES 2000: 1: 245251. Monografias Tercer Milenio, Sociedad Entomológica Aragonesa, Zaragoza, España.

ELGUETA M (2000) Coleoptera de Chile. En: MartínPiera F, J J Morrone \& A melic (eds) Hacia un proyecto CYTED. PrIBES 2000: 1: 145-154. Monografías Tercer Milenio, Sociedad Entomológica Aragonesa, Zaragoza, España.

ELGUETA M, A CAMOUSSEIGHT \& CS CARBONELL (1999) Catálogo de Orthoptera de Chile. Publicación Ocasional Museo Nacional de Historia Natural, Chile 54: 5- 60.

FEUILLEÉ LE (1714-1725) Journal des observations physiques, mathématiques et botaniques. Faites par l'ordre du Roy sur les côtes orientales de 1'Amérique meridionale \& dans les Indes Occidentales, depuis l'année 1707 jusques en 1712. Paris, 3 vols. Editorial Pierre Giffart. vol. I (1-504), vol. II (503-767), vol. III (1-426).

GAY C (1845-1854) Historia física y política de Chile según documentos adquiridos en esta república durante doce años de residencia en ella y publicada bajo los auspicios del Supremo Gobierno. Botánica (Flora chilena). Santiago, París. 8 vols., 1 Atlas. Editorial Faine \& Thunot, vol. I (1-496), vol. II (1-
534), vol. III (1-484), vol. IV (1-516), vol. V (1$479)$, vol. VI (1-551), vol. VII (1-515), vol. VIII (1448).

GODFRAY HCJ (2002) Challenges for taxonomy: the discipline will have to reinvent itself if it is to survive and flourish. Nature 417: 17-19.

GONZÁLEZ CR (1995) Diptera. En: Simonetti JA, MT Kalin-Arroyo, AE Spotorno \& E Lozada (eds) Diversidad biológica de Chile: 256-263. Comisión Nacional de Investigación Científica y Tecnológica, Santiago, Chile.

GONZÁLEZ E (2003) The freshwater amphipods Hyalella Smith, 1874 in Chile (Crustacea: Amphipoda). Revista Chilena de Historia Natural 76: 623-637.

GONZÁLEZ ER \& M THIEL (2004) Indice bibliográfico sobre biodiversidad acuática de Chile: Crustáceos Peracáridos (Crustacea). Ciencia y Tecnología Marina 27: 133-150.

GOTSCHLICH B (1904) Biografía del Dr. Rodulfo Amando Philippi (1808-1904). Imprenta Central J. Lampert, Valdivia, Chile. 185 pp.

GUNCKEL H (1953) Plantas chilenas estudiadas por Linneo. Revista Universitaria (Chile) 38: 67-76.

HE A (1998) Checklist of the mosses of Chile. Journal of the Hattori Botanical Laboratory (Japón) 85: 103 189.

HUNT D (1999) CITES Cactaceae checklist. Royal Botanical Gardens Kew \& International Organization for Succulent Plant Study, Kew. 315 pp.

JAKSIC FM \& I LAZO (1994) La contribución de Darwin al conocimiento de los vertebrados terrestres de Chile. Revista Chilena de Historia Natural 67: 9-26.

LANE MA (1996) Roles of natural history collection. Annals of the Missouri Botanical Garden 83: 536545 .

LARRAÍN A (1995) Biodiversidad de equinodermos chilenos: estado actual del conocimiento y sinopsis biosistemática. Gayana Zoología 59: 73-96.

LAZO W (2001) Hongos de Chile: atlas micológico. Facultad de Ciencias, Universidad de Chile. Salecianos S.A., Santiago, Chile. 231 pp.

LETELIER S, MA VEGA, AM RAMOS \& E CARREÑO (2003) Base de datos del Museo Nacional de Historia Natural: moluscos de Chile. Revista de Biología Tropical 51: 33-137.

LINNEO C (1753) Species plantarum. Holmiae, Stockholm. 2 vols. Suecia, vol. I (1560), vol. II (561-1200).

LOOSER G (1931) Los primeros trabajos de historia natural publicados en Chile. A la memoria de Carlos Bertero en el centenario de su muerte. Boletín de la Biblioteca Nacional, Chile 2: 17-19.

LOOSER G (1953) Plantas chilenas estudiadas por Linneo. Revista Universitaria, Chile 38: 67-76.

LOZADA E \& J SOLERVICENS (1995) Invertebrados de Chile. En Simonetti JA, MT Kalin-Arroyo, AE Spotorno \& E Lozada (eds) Diversidad biológica de Chile: 90-92. CONICYT, Santiago, Chile.

MÁRQUEZ-BRETON B (1982) Orígenes del darwinismo en Chile. Editorial Andrés Bello. Santiago, Chile. $111 \mathrm{pp}$.

MARTICORENA C \& M QUEZADA (1985) Catálogo de la flora vascular de Chile. Gayana Botánica 42: 5157.

MARTICORENA C \& M QUEZADA (1987) Adiciones a la flora de Chile. Gayana Botánica 44: 39-44.

MARTICORENA C \& R RODRÍGUEZ (eds) (1995) Flora de Chile. Volumen I. Pteridophyta-Gymnospermae. Universidad de Concepción, Concepción, Chile. $351 \mathrm{pp}$. 
MARTICORENA C \& R RODRÍGUEZ (eds) (2001) Flora de Chile. Volumen 2. Winteraceae-Ranunculaceae. Universidad de Concepción, Concepción, Chile. 99 pp.

MARTICORENA C (1995) Historia de la exploración botánica en Chile. En: Marticorena C \& R Rodríguez (eds) Flora de Chile. Volumen I: 1-62. Pteridophyta-Gymnospermae. Universidad de Concepción, Concepción, Chile.

MARTICORENA C (1996) Bibliografía botánica taxonómica de Chile. Gayana Botánica 53: 1-263.

MAY RM (1988) How many species are there on Earth? Science 241: 1441-1449.

MENZIES RJ (1961) The zoogeography, ecology, and systematics of the chilean marine isopods. Reports of the Lund University Chile expedition 1948-1949. Lunds Universitets Arsskrift Ny Foljd, 162 pp.

MOLINA JI (1782) Saggio sulla Storia Naturale et Civile del Chili. Bologna, Italia. 367 pp.

MORONI J \& A CAMOUSSEIGHT (1976) Aporte al estudio de las baratas de Chile. Boletín de la Sociedad de Biología de Concepción (Chile) 50:4351.

MOSTNY-GLASER G \& H NIEMEYER-FERNÁNDEZ (1983) Museo Nacional de Historia Natural. Dirección de Bibliotecas, Archivos y Museos. Ministerio de Educación Pública, Santiago, Chile. $143 \mathrm{pp}$.

MOYANO GHI (1991) Bryozoa marinos chilenos VIII: una síntesis zoogeográfica con consideraciones sistemáticas y la descripción de diez especies y dos géneros nuevos. Gayana Zoología 55: 305-389.

MUJICA F \& C VERGARA (1980) Flora fungosa chilena. Segunda Edición. Ciencias Agrícolas, Facultad de Agronomía, Universidad de Chile, Chile 5: 1-308.

MUÑOZ-PIZARRO C (1960) Las especies de plantas descritas por R. A. Philippi en el siglo XIX. Estudio crítico en la identificación de sus tipos nomenclaturales. Ediciones Universidad de Chile, Santiago, Chile. 189 pp.

MUÑOZ-SCHICK M (2003) 100 años de la sección botánica del Museo Nacional de Historia Natural (1889-1989). Boletín del Museo Nacional de Historia Natural (Chile) 42: 181-202.

MUÑOZ-SCHICK M (2003) Importancia de los Philippi en el desarrollo de la botánica chilena. En: Larroucau $M$ (ed) El orden prodigioso del mundo natural. Rudolph Amandus Philippi: 41-51. Universidad Austral de Chile y Pehuén Editores, Valdivia, Chile.

NÚÑEZ H \& FM JAKSIC (1992) Lista comentada de reptiles terrestres de Chile continental. Boletín del Museo Nacional de Historia Natural (Chile) 43: 6391.

PARRA LE (1995) Lepidoptera. En: Simonetti JA, MT Kalin-Arroyo, AE Spotorno \& E Lozada (eds.) Diversidad biológica de Chile: 269-279. CONICYT, Santiago, Chile.

PEÑAILILLO P (1998) Nuevas combinaciones en el género Nassella E. Desv. emend. Barkworth (1990) (Poaceae, Stipeae). Gayana Botánica 55: 85-88.

PEQUEÑO G (1989) Peces de Chile. Lista sistemática renovada y completada. Revista de Biología Marina (Chile) 24: 1-132

PEQUEÑO G (1997) Peces de Chile. Lista sistemática revisada y comentada: Addendum. Revista de Biología Marina (Valparaíso) 32: 77-94.

PEQUEÑO G (2003) Aporte de Rudolph A. Philippi a la zoología. En Larroucau M (ed) El orden prodigioso del mundo natural. Rudolph Amandus Philippi: 29-
40. Universidad Austral de Chile y Pehuén Editores, Valdivia, Chile.

PHILIPPI RA (1862) Sobre algunos insectos de Magallánes. Anales de la Universidad de Chile 21: 407-416.

PHILIPPI RA (1867) Sobre las plantas descritas por el padre Feuillée. Anales de la Universidad de Chile 29: 760-775.

PHILIPPI RA (1869) Elementos de botánica. Imprenta Nacional, Santiago de Chile, Chile. 571 pp.

PHILIPPI RA (1875) Sobre las plantas que Chile posee en común con Europa, por el doctor don Rodulfo A. Philippi. Anales de la Universidad de Chile 47: 131-140

PHILIPPI RA (1885) Elementos de historia natural. Cuarta edición. Imprenta FA Brockhaus, Leipzig. Santiago de Chile. 410 pp.

PHILIPPI RA (1892) Los zoófitos chilenos. Anales del Museo Nacional de Chile 7: 5-11.

PHILIPPI RA (1902) Figuras i descripción de aves chilenas. Anales del Museo Nacional de Chile 15: $1-110$.

PORTER CE (1902) Galería de naturalistas de Chile: Dr R. A. Philippi. Revista Chilena de Historia Natural 6: 189.

PORTER CE (1904) Dr. R. A. Philippi. 1808-1904. Revista Chilena de Historia Natural 8: 173.

PRADO E (1995) Hemiptera - Heteroptera. En: Simonetti JA, MT Kalin-Arroyo, AE Spotorno \& E Lozada (eds) Diversidad biológica de Chile: 241-245. CONICYT, Santiago, Chile.

REICHARDT H (1968a) Catalogue of New World Dermaptera (Insecta). Part I: Introduction and Pygudicranoidea. Papéis Avulsos Zoologia São Paulo 21: 183-193

REICHARDT H (1968b) Catalogue of New World Dermaptera (Insecta). Part II: Labioidea, Carcinophoridae. Papéis Avulsos Zoologia São Paulo 22: 35-46.

RUIZ R \& N BAHAMONDE (1989) Cladóceros y copépodos límnicos en Chile y su distribución geográfica. Lista sistemática. Publicación Ocasional, Museo Nacional de Historia Natural, Chile 45: 5-48.

SALDIVIA Z (2003) La visión de la naturaleza en tres científicos del siglo XIX: Gay, Domeyko y Philippi. Ed. Universidad de Santiago de Chile, Santiago, $197 \mathrm{pp}$.

SERRANO S (1993) Universidad y nación: Chile en el siglo XIX. Editorial Universitaria, Santiago, Chile. $276 \mathrm{pp}$.

SHAFER HB, RN FISHER \& C DAVIDSON (1998) The role of natural history collections in documenting species declines. Trends in Ecology and Evolution 13: 27-30.

SIGÜENZA AN \& J LLORENTE-BOUSQUETS (1994) Museos y la conservación de la biodiversidad. En: Llonrente-Bousquets J \& IL Vega (eds) Taxonomía biológica: 229-257. Universidad Nacional Autónoma de México, México.

SIMONETTI JA, MT KALIN-ARROYO, AE SPOTORNO \& E LOZADA (eds.) (1995) Diversidad biológica de Chile. Comisión Nacional de Investigación Científica y Tecnológica, Santiago, Chile. 364 pp.

STEENBUCK U (2003) Rudolph Amandus Philippi (18081904): vida y obra. En: Larroucau M (ed) El orden prodigioso del mundo natural. Rudolph Amandus Philippi: 11-28. Universidad Austral de Chile y Pehuén Editores, Valdivia, Chile.

TAMAYO M, H NÚÑ̃EZ \& J YÁÑEZ (1987) Lista 
sistemática actualizada de los mamíferos vivientes en Chile y sus nombres comunes. Noticiario Mensual Museo Nacional de Historia Natural 312: $1-13$.

TAYLOR CM \& M MUÑOZ (1994) The botanical works of Philippi, father and son, in Chile. Annals of the Missouri Botanical Garden 81: 743-748.

VALDOVINOS-ZARGES C (1999) Biodiversidad de moluscos chilenos: base de datos taxonómica y distribucional. Gayana Zoología 63: 111-164.

VELOSO A \& J NAVARRO (1988) Lista sistemática y distribución de anfibios y reptiles de Chile. Bolletino del Museo Regionale di Scienze Natturali, Torino 6: 481-539

YÁÑEZ J \& A MUÑOZ-PEDREROS (2000) Mamíferos vivientes de Chile. En: Muñoz-Pedreros A \& J Yáñez-Valenzuela (eds) Mamíferos de Chile: 37-40. CEA Ediciones, Valdivia, Chile.

ZIZKA G (1992) Observaciones sobre la historia de la investigación botánica en Chile. En: J Grau \& G Zizka (eds) Flora Silvestre de Chile: 19: 25-30. Palmengarten Sonderheft, Frankfurt.

\section{ANEXO}

Catálogo de publicaciones de Philippi sobre insectos

\section{Catalogue of Philippi's publications on insects}

PHILIPPI RA (1859a) En: PHILIPPI F Algunas especies nuevas de Coleópteros de la provincia de Valdivia. Anales de la Universidad de Chile 16: 656-678.

PHILIPPI RA (1859b) Descripción de tres especies nuevas de Coleópteros chilenos. Anales de la Universidad de Chile 16: 1085-1088.

PHILIPPI RA (1859c) Descripción de algunas nuevas especies de Mariposas chilenas, principalmente de la provincia de Valdivia. Anales de la Universidad de Chile 16: 1088-1114.

PHILIPPI RA (1860a) Viage al Desierto de Atacama. Ed. Librería de Eduardo Anton, Halle, Sajonia, 174 pp.

PHILIPPI RA (1860b) Beschreibung einiger neuer Chilenischer Schmetterlinge. Linnae Ent. Berlin 16: 265-297.

PHILIPPI RA (1861a) Comunicaciones de don Rodulfo Amando Philippi a las Facultades de Ciencias Físicas i de Medicina, leidas en la sesion que juntas celebraron el 12 de junio de 1861. III Descripcion de una nueva especie de mosca. Anales de la Universidad de Chile 18: 724-731.

PHILIPPI RA (1861b) En: PHILIPPI F Observaciones sobre los Lamelicornios de Chile, descritos en la obra del Señor Gay, con descripción de algunas especies nuevas. Anales de la Universidad de Chile 18: 735-744.

PHILIPPI RA (1861c) Beschreibung einer neuen Fliege, deren Laven in der Nase und Stirnhôhle einer Frau gelebt haben. Zeitschrift fûr die Gesammten Naturwissenschaften 17: 513-515.

PHILIPPI RA (1862a) Viaje a los baños i al nuevo volcan de Chillán. Anales de la Universidad de Chile 21: 377-389.
PHILIPPI RA (1862b) Sobre algunos insectos de Magallanes. Anales de la Universidad de Chile 21: 377-389.

PHILIPPI RA (1863a) Catálogo de los insectos recojidos. En: COX G, Jeografia de Chile. Viaje a las rejiones septentrionales de la Patagonia. Anales de la Universidad de Chile 23: 476-478.

PHILIPPI RA (1863b) Zwei neue Käfer aus Chile. Entomologische Zeitung 24:132-135.

PHILIPPI RA (1863c) Verzeichniss der im Museum von Santiago befindlichen Chilenischen Orthopteren. Zeitschrift für die Gesammten Naturwissenschaften 21: 217-245

PHILIPPI RA (1863d) Beschreibung einer neuen Acridioide aus der Argentinischen Republik. Zeitschrift für die Gesammten Naturwissenschaften 21: 444-447.

PHILIPPI RA (1864a) Eudelia rufescens Ph., ein neuer Spinner von Chile. Entomologische Zeitung 25: 9196.

PHILIPPI RA (1864b) Coleopterodes Philippi ein neues Geschlecht der Wanzen. Entomologische Zeitung 25: 306-308.

PHILIPPI RA (1864c) Sobre algunos Coleópteros nuevos de Chile de la familia de las Melolontideas. Anales de la Universidad de Chile 24: 435-462.

PHILIPPI RA (1864d) En: RONDANI C Diperorum species et genera aliqua exotica revista annotatonovis numullis descriptis. Arch. Per. La Zool., L Anat. E la Fisiol Modena 3: 23-99.

PHILIPPI RA (1865a) Descripción de algunos insectos nuevos chilenos. Anales de la Universidad de Chile 26: 651-660.

PHILIPPI RA (1865b) Acanthia valdiviana und Bacteria unifoliata. Entomologische Zeitung 26: 63-65.

PHILIPPI RA (1865c) Aufzählung der chilenischen Dipteren. Verhandlungen der kaiserlich-königlichen zoologisch-botanischen Gesellschaft in Wien 15 : 595.782. pls. 23-29.

PHILIPPI RA (1866) Einige Insekten von Chile. Entomologische Zeitung 27: 109-117.

PHILIPPI RA (1870) Descripción de una nueva mariposa chilena del jénero Erebus. Anales de la Universidad de Chile 36: 213-215.

PHILIPPI RA (1871a) Esploración científica practicada por órden del Supremo gobierno i según las instrucciones del Doctor don R.A. Philippi, por don Carlos Juliet, ayudante de la Comision esploradora del mar i costas de Chiloé i Llanquihue, a bordo del "Covadonga". Anales de la Universidad de Chile 39:126-156.

PHILIPPI RA (1871b) Beschreibung einiger neuer chilenischer Insekten. Entomologische Zeitung 32: 285-295.

PHILIPPI RA (1873) Chilenische Insekten. Entomologische Zeitung 34: 296-316.

PHILIPPI RA (1875) Descripcion de un nuevo díptero chileno. Anales de la Universidad de Chile 47: 83-86.

PHILIPPI RA \& AHE PHILIPPI (1860) Coleoptera nonnulla nova Chilensia preaesertim Valdivia. Entomologische Zeitung 21: 245-251.

PHILIPPI RA \& F PHILIPPI (1864a) Beschreibung einiger neuen Chilenischen Käfer. Entomologische Zeitung 25: 266-284.

PHILIPPI RA \& F PHILIPPI (1864b) Beschreibung einiger neuen Chilenischen Käfer. Entomologische Zeitung 25: 313-406. 